\title{
The whole crack singular element for 2-D boundary element analysis of multiple straight cracks in the general anisotropic solids
}

\author{
M. Denda and E. Mattingly \\ Rutgers University, Mech. \& Aero. Engng. Dept. \\ Piscataway, NJ 08854-8058, U.S.A.
}

\begin{abstract}
We develop a singular crack element for the general anisotropic solids in twodimensions for the mixed mode boundary element analysis of multiple straight cracks. Given a normalized crack along an interval $(-1,+1)$ on the $X$-axis, we represent the crack opening displacement (COD) by the continuous distribution of dislocation dipoles, which is interpolated by the Chebyshev polynomials with the $\sqrt{1-X^{2}}$ weight function. The analytical integration of the dislocation dipole distribution leads to a closed form displacement formula for the crack with the $\sqrt{r}$ COD and the $1 / \sqrt{r}$ stress singularity at its tips. In the BE solution, the stress intensity factors are determined, along with the unknown boundary displacements and tractions, without the post-processing. The proposed crack element, called the whole crack singular element (WCSE), drastically simplifies the mixed mode analysis of multiple straight cracks in the general anisotropic solids with no sacrifice of the accuracy.
\end{abstract}

\section{Introduction}

The BEM papers for the two-dimensional crack analysis in the anisotropic solids deal with the plane strain/stress (Sollero and Aliabadi [1], Denda [2]) and the generalized plane strain (Ang and Clements [3], Berger and Tewary [4], Tan et al.[5], Denda [6]). In the generalized plane strain the coupling of the in-plane and the out-of-plane deformations exists. While Denda [6] has addressed the full coupling of the Mode I, II, and III stress intensity factors in the generalized plane strain, all others dealt with simpler cases of decoupled anisotropy. The crack analysis for the materials in the crystal classes of triclinic, monoclinic and trigonal systems requires such a coupling since they do not have a plane of symmetry normal to the $x_{3}$-axis (i.e., the out-of-plane axis). The other crystals which have this symmetry also exhibit the coupling if their crystal axes are rotated out of the symmetry position.

The modeling of the crack tip singularity is the central issue of the crack analysis in anisotropic elasticity. Tan and Gao [7] adopted the quarter-point traction and displacement crack tip elements and derived analytical expressions for the stress intensity factors given by the nodal traction and displacement of these elements. Sollero and Aliabadi [1], in the dual boundary element method, used the J-integral and the ratio of the crack opening displacements near the crack tip. Snyder and Cruse [8] used the Green's function for the single crack and calculated the 
stress intensity factors analytically without modeling the crack surface. Denda $[2,6]$, modeling the crack by the continuous distribution of dislocation dipoles, adopted the conservation integral developed by Chen and Shield [9] to calculate stress intensity factors. Although the accuracy of stress intensity results by the conservation integral is impressive, the post-processing requirement of the contour integral evaluation around each crack tip is an extra burden for the multiple cracks. The objective of this paper is to get rid of this post-processing requirement for the stress intensity factor evaluation by developing a singular crack element for straight cracks. Such a singular crack element was developed by Denda and Dong [10] for the isotropic solids, but not available for the general anisotropic solids.

In a normalized local coordinate system attached along a straight crack $-1 \leq$ $X \leq+1$ on the $X$-axis, we represent the crack opening displacement (COD) by the continuous distribution of dislocation dipoles, which is interpolated by the Chebyshev polynomials with the $\sqrt{1-X^{2}}$ weight function. We evaluate the dislocation dipole distribution analytically to derive a closed form displacement formula for the crack with the $\sqrt{r}$ COD and the $1 / \sqrt{r}$ stress singularity at its tips, where $r$ is the distance from the crack tip. Since the crack opening displacement over the whole crack length is interpolated by the above scheme, the element is called the whole crack singular element (WCSE). In the BE solution, the stress intensity factors are determined, along with the unknown boundary displacements and tractions, without the post-processing. The numerical results demonstrate that the proposed WCSE drastically simplifies the mixed mode analysis of multiple straight cracks in the general anisotropic solids with no sacrifice of the accuracy.

\section{Basic Equations in Generalized Plane Strain}

In the generalized plane strain anisotropic elasticity problems the displacement components $u_{i}(i=1,2,3)$ depend only on two coordinates $x_{1}$ and $x_{2}$. The non zero strain components are given by

$$
e_{1}=\frac{\partial u_{1}}{\partial x_{1}}, \quad e_{2}=\frac{\partial u_{2}}{\partial x_{2}}, \quad e_{4}=\frac{\partial u_{3}}{\partial x_{2}}, \quad e_{5}=\frac{\partial u_{3}}{\partial x_{1}}, \quad e_{6}=\frac{\partial u_{2}}{\partial x_{1}}+\frac{\partial u_{1}}{\partial x_{2}},
$$

where a single suffix $M$ attached to the strain components replaces a pair of suffices $i j$ following the convention $(11 \rightarrow 1),(22 \rightarrow 2),(33 \rightarrow 3),(23 \rightarrow 4),(31 \rightarrow 5$, $(12 \rightarrow 6)$. Similar suffix convention is used for the components of the stress $\left(\sigma_{M}\right)$ and the compliance $\left(s_{M N}\right)$ whenever convenient. We consider the anisotropic material arranged such that it does not have a symmetry plane parallel to the $x_{1} x_{2}$-plane. The strain-stress relations for such material are given by

$$
e_{M}=\sum_{N=1}^{6} S_{M N} \sigma_{N} \quad(\mathrm{M}, \mathrm{N}=1,2,4,5,6),
$$

where $S_{M N}$ is the reduced compliance defined by

$$
S_{M N}=s_{M N}-\left(s_{M 3} s_{3 N}\right) / s_{33} \quad(\mathrm{M}, \mathrm{N}=1,2,4,5,6)
$$


in terms of the 3 -D compliance constants $s_{M N}(M, N=1,2,3,4,5,6)$. In this paper the summation over an index is indicated explicitly without using the summation convention for the repeated indices.

Lekhnitskii [11] and Eshelby et al. [12] have shown that the displacement $u_{i}$ and the stress function $\phi_{i}$ are given in the form,

$$
u_{i}=2 \Re \sum_{\alpha=1}^{3} A_{i \alpha} f_{\alpha}\left(z_{\alpha}\right), \quad \phi_{i}=2 \Re \sum_{\alpha=1}^{3} L_{i \alpha} f_{\alpha}\left(z_{\alpha}\right)
$$

in terms of three functions $f_{1}\left(z_{1}\right), f_{2}\left(z_{2}\right), f_{3}\left(z_{3}\right)$, each of which is analytic in its argument $z_{\alpha}=x_{1}+p_{\alpha} x_{2}$. Here $p_{\alpha}$ are three distinct complex numbers which, along with their three conjugates, are the roots of the sixth-order polynomial characteristic equations in $p$,

$$
d^{(4)}(p) d^{(2)}(p)-d^{(3)}(p) d^{(3)}(p)=0
$$

where

$$
\begin{gathered}
d^{(4)}(p)=p^{4} S_{11}-2 p^{3} S_{16}+p^{2}\left(2 S_{12}+S_{66}\right)-2 p S_{26}+S_{22}, \\
d^{(3)}(p)=p^{3} S_{15}-p^{2}\left(S_{14}+S_{56}\right)+p\left(S_{25}+S_{46}\right)-S_{24}, \\
d^{(2)}(p)=p^{2} S_{55}-2 p S_{45}+S_{44} .
\end{gathered}
$$

The symbol $\Re$ indicates the real part of a complex variable. The coefficients $L_{i \alpha}$ and $A_{i \alpha}$ are the components of $3 \times 3$ matrices $\mathbf{L}$ and $\mathbf{A}$ defined by

$$
\mathbf{L}=\left[\mathbf{l}_{\mathbf{1}}, \mathbf{l}_{\mathbf{2}}, \mathbf{l}_{\mathbf{3}},\right]=\left[\begin{array}{ccc}
-p_{1} L_{21} & -p_{2} L_{22} & -p_{3} l_{3} L_{33} \\
L_{21} & L_{22} & l_{3} L_{33} \\
l_{1} L_{21} & l_{2} L_{22} & L_{33}
\end{array}\right]
$$

where

$$
l_{\alpha}=\frac{d^{(3)}\left(p_{\alpha}\right)}{d^{(2)}\left(p_{\alpha}\right)} \quad(\alpha=1,2), \quad l_{3}=\frac{d^{(3)}\left(p_{3}\right)}{d^{(4)}\left(p_{3}\right)}
$$

and

$$
\mathbf{A}=\left[\mathbf{a}_{1}, \mathbf{a}_{2}, \mathbf{a}_{3}\right]
$$

with

$$
\mathbf{a}_{\alpha}=\left\{\begin{array}{c}
A_{1 \alpha} \\
A_{2 \alpha} \\
A_{3 \alpha}
\end{array}\right\}=\left[\begin{array}{ccc}
s_{16}-s_{11} p_{\alpha}, & s_{12}, & s_{14}-s_{15} p_{\alpha} \\
\frac{s_{26}-s_{21} p_{\alpha}}{p_{\alpha}}, & \frac{s_{22}}{p_{\alpha}}, & \frac{s_{24}-s_{25} p_{\alpha}}{p_{\alpha}} \\
s_{56}-s_{51} p_{\alpha}, & s_{52}, & s_{54}-s_{55} p_{\alpha}
\end{array}\right]\left\{\begin{array}{l}
L_{1 \alpha} \\
L_{2 \alpha} \\
L_{3 \alpha}
\end{array}\right\} .
$$

For each characteristic root $p_{\alpha}$ we can determine vectors $\mathbf{l}_{\alpha}$ and $\mathbf{a}_{\alpha}$ up to an arbitrary multiplying factor. Following the details provided by Denda [6], we normalize the problem by

$$
2 \sum_{i=1}^{3} L_{i \alpha} A_{i \alpha}=1 \quad(\alpha=1,2,3) .
$$

Finally, the stress components $\sigma_{i j}$ are given by

$$
\sigma_{1 i}=-\frac{\partial \phi_{i}}{\partial x_{2}}, \quad \sigma_{2 i}=\frac{\partial \phi_{i}}{\partial x_{1}} .
$$




\section{Direct Formulation of the BEM}

We use two fundamental solutions in our BEM formulation: the line fore and the dislocation dipole. The dislocation dipole is an infinitesimal segment $\left(d \eta_{1}, d \eta_{2}\right)$ of length $d s$ over which a displacement jump is prescribed. Consider a line force in $x_{k}$ direction at $\left(\eta_{1}, \eta_{2}\right)$; the resulting displacement component in the $x_{j}$ direction at $\left(x_{1}, x_{2}\right)$ is given by

$$
G_{j k}\left(x_{1}, x_{2} ; \eta_{1}, \eta_{2}\right)=\Im \frac{1}{\pi} \sum_{\alpha=1}^{3} A_{j \alpha} A_{k \alpha} \ln \left(z_{\alpha}-\xi_{\alpha}\right),
$$

where $z_{\alpha}=x_{1}+p_{\alpha} x_{2}$ and $\xi_{\alpha}=\eta_{1}+p_{\alpha} \eta_{2}(\alpha=1,2,3)$ and $\Im$ is the imaginary part of a complex variable. Consider a dislocation dipole at $\left(\eta_{1}, \eta_{2}\right)$ in $x_{k}$ direction; the resulting displacement component in $x_{j}$ direction at $\left(x_{1}, x_{2}\right)$ is given by

$$
G_{j k}^{(d)}\left(x_{1}, x_{2} ; \eta_{1}, \eta_{2}\right) d s=-\Im \frac{1}{\pi} \sum_{\alpha=1}^{3} A_{j \alpha} L_{k \alpha} \frac{d \xi_{\alpha}}{z_{\alpha}-\xi_{\alpha}}
$$

where $d \xi_{\alpha}=d \eta_{1}+p_{\alpha} d \eta_{2}$. See Denda [6] for detailed derivation of these solutions.

Consider a finite domain $R$ with the boundary $\partial R$ with the boundary displacement $u_{j}$ and the traction $t_{j}$. The displacement in $R$ is given by the distributions of point forces and dislocation dipoles with the magnitudes $t_{j}$ and $u_{j}$, respectively, over the closed contour $\partial R$ in the infinite domain. This is a physical interpretation of Somigliana's identity (Denda [6], Altiero and Gavazza [13], Eshelby [14]) which is used in the direct formulation of the BEM.

We discretize and approximate the original boundary by a set of straight lines. The boundary integrals for the point force and the dislocation dipole distributions are evaluated analytically using the quadratic interpolation function for the boundary displacement and traction. The explicit formulas for the displacement, displacement gradient, stress and the traction for generalized plane strain can be found in [6]. Since the boundary integrals are evaluated analytically, there is no need to deal with the singular and the hypersingular integrals. The boundary equations are all algebraic rather than integral equations. Otherwise we follow the standard procedure of the BEM implementation as discussed by Denda [6].

\section{Whole Crack Singular Element}

A crack $L$ with the crack opening displacement $\delta_{k}$ of a traction-free crack in an infinite body is represented by the continuous distribution of the dislocation dipoles with the magnitude $\delta_{k}$ (Denda [6]). The displacement due to the crack is given by the integral of (13),

$$
u_{j}^{(d)}\left(x_{1}, x_{2}\right)=-\Im \frac{1}{\pi} \int_{L} \sum_{\alpha=1}^{3} A_{j \alpha} \sum_{k=1}^{3} L_{k \alpha} \delta_{k} \frac{d \xi_{\alpha}}{z_{\alpha}-\xi_{\alpha}},
$$


along the crack, where $\xi_{\alpha}=\eta_{1}+p_{\alpha} \eta_{2}$. Consider a straight crack in the interval $(-1,+1)$ on the horizontal coordinate axis where $\xi_{\alpha}=\eta_{1}$ for all values of $\alpha$ $(=1,2,3)$ since $\eta_{2}=0$. Simply denote $\eta=\eta_{1}$ by omitting the subscript and rewrite (14) as

$$
u_{j}^{(d)}\left(x_{1}, x_{2}\right)=\Im \frac{1}{\pi} \int_{-1}^{+1} \sum_{\alpha=1}^{3} A_{j \alpha} \sum_{k=1}^{3} L_{k \alpha} \delta_{k}(\eta) \frac{d \eta}{\eta-z_{\alpha}} .
$$

Expecting the $\sqrt{r}$ behavior of the crack opening displacement components at the crack tips we interpolate them by

$$
\delta_{k}(\eta)=\sqrt{1-\eta^{2}} \sum_{m=1}^{M} \delta_{k}^{(m)} U_{m-1}(\eta),
$$

where $U_{m-1}(\eta)$ is Chebyshev polynomial of the second kind. Substitute (16) into (15) and evaluate the integral analytically to get

$$
u_{j}^{(d)}\left(x_{1}, x_{2}\right)=-\Im \sum_{m=1}^{M} \sum_{\alpha=1}^{3} A_{j \alpha} \sum_{k=1}^{3} L_{k \alpha} \delta_{k}^{(m)} R_{m}\left(z_{\alpha}\right)
$$

where

$$
R_{m}\left(z_{\alpha}\right)=\left(z_{\alpha}-\sqrt{\left(z_{\alpha}\right)^{2}-1}\right)^{m} \quad(m \geq 1) .
$$

Similarly, the stress function is given by

$$
\phi_{j}^{(d)}\left(x_{1}, x_{2}\right)=-\Im \sum_{m=1}^{M} \sum_{\alpha=1}^{3} L_{j \alpha} \sum_{k=1}^{3} L_{k \alpha} \delta_{k}^{(m)} R_{m}\left(z_{\alpha}\right) .
$$

When the half-crack length is $a$, instead of 1 , the formulas (17) and (19) still apply if we replace the arguments $z_{\alpha}$ by the normalized arguments $Z_{\alpha}=z_{\alpha} / a$ such as

$$
\begin{aligned}
u_{j}^{(d)}\left(x_{1}, x_{2}\right) & =-\Im \sum_{m=1}^{M} \sum_{\alpha=1}^{3} A_{j \alpha} \sum_{k=1}^{3} L_{k \alpha} \delta_{k}^{(m)} R_{m}\left(Z_{\alpha}\right), \\
\phi_{j}^{(d)}\left(x_{1}, x_{2}\right) & =-\Im \sum_{m=1}^{M} \sum_{\alpha=1}^{3} L_{j \alpha} \sum_{k=1}^{3} L_{k \alpha} \delta_{k}^{(m)} R_{m}\left(Z_{\alpha}\right) .
\end{aligned}
$$

The stress components are obtained by substituting (20) into (11) with the result

$$
\begin{aligned}
\sigma_{2 j}^{(d)}\left(x_{1}, x_{2}\right) & =-\frac{1}{a} \Im \sum_{m=1}^{M} \sum_{\alpha=1}^{3} L_{j \alpha} \sum_{k=1}^{3} L_{k \alpha} \delta_{k}^{(m)} m G_{m-1}\left(Z_{\alpha}\right), \\
\sigma_{1 j}^{(d)}\left(x_{1}, x_{2}\right) & =\frac{1}{a} \Im \sum_{m=1}^{M} \sum_{\alpha=1}^{3} p_{\alpha} L_{j \alpha} \sum_{k=1}^{3} L_{k \alpha} \delta_{k}^{(m)} m G_{m-1}\left(Z_{\alpha}\right),
\end{aligned}
$$


where

$$
G^{(m-1)}\left(Z_{\alpha}\right)=-\frac{\left(Z_{\alpha}-\sqrt{\left(Z_{\alpha}\right)^{2}-1}\right)^{m}}{\sqrt{\left(Z_{\alpha}\right)^{2}-1}} \quad(m \geq 1) .
$$

By investigating the limiting behavior on the crack line we find

$$
\left(t_{j}\right)^{ \pm}(X)= \pm \frac{1}{a} \Im \sum_{m=1}^{M} \sum_{\alpha=1}^{3} L_{j \alpha} \sum_{k=1}^{3} L_{k \alpha} \delta_{k}^{(m)} m U_{m-1}(X) \quad(|X| \leq 1),
$$

where $X=x_{1} / a$ and the superscripts + and - indicate limits from above and below the horizontal coordinate axis, respectively. From the limiting behavior in front of each crack tip at $X= \pm 1$ we find

$$
K_{j}( \pm 1)=\sqrt{\frac{\pi}{a}} \Im \sum_{m=1}^{M}( \pm)^{m+1} \sum_{\alpha=1}^{3} L_{j \alpha} \sum_{k=1}^{3} L_{k \alpha} \delta_{k}^{(m)} m
$$

which give Mode I $\left(K_{I}=K_{2}\right)$, Mode II $\left(K_{I I}=K_{1}\right)$ and Mode III $\left(K_{I I I}=K_{3}\right)$ stress intensity factors.

When the crack, centered at the origin, is inclined we introduce a pair of rotated local coordinate axes parallel and perpendicular to the crack. The formulas (20)-(24) still apply if we use the compliance, characteristic roots, and matrices $\mathbf{L}$ and $\mathbf{A}$ in the rotated local coordinate system. When the crack is not centered at the origin we need to introduce a pair of translated local coordinate axes located at the center of the crack. Under this translation, if not accompanied by the rotation, the formulas (20)-(24) can be applied without modification.

\section{Multiple Crack Analysis}

Consider $\mathrm{N}$ straight cracks in a finite body. Each crack has its own local coordinate system with its origin at the crack center and the horizontal axis along the crack. We interpolate the COD of each crack by M Chebyshev polynomials in (16); the number $M$ can be different for each crack. This introduces a $3 M$ - dimensional COD vector $\{\delta\}_{\mathbf{n}}(n=1,2, \cdots, N)$ for each crack, which can be determined by evaluating the traction at $M$ collocation points on its own crack surface. Unlike the quadrature formula for the singular integrals, where the selection of quadrature and collocation points must strictly obey a certain rule, our selection of the collocation point on the crack surface is arbitrary. The best result is obtained by including the crack tips among the collocation points; as seen from (23), the traction on the crack surface is bounded at the crack tip allowing its evaluation. Contrast this with the numerical quadrature that must avoid the crack tip as a collocation point. A $3 M$-dimensional global traction equation for crack $r$ has the form

$$
\{\mathbf{s}\}_{\mathbf{r}}=[\mathbf{H}]_{\mathbf{r}}^{*}\{\mathbf{u}\}+[\mathbf{G}]_{\mathbf{r}}^{*}\{\mathbf{t}\}+\sum_{\mathbf{n}=\mathbf{1}}^{\mathbf{N}}[\mathbf{D}]_{\mathbf{r n}}^{*}\{\delta\}_{\mathbf{n}}=\{\mathbf{0}\} \quad(\mathbf{r}=\mathbf{1}, \mathbf{2}, \cdots, \mathbf{N})
$$


which is set to zero for the traction-free crack. The contributions from the boundary displacement $\{\mathbf{u}\}$ and the traction $\{\mathbf{t}\}$ are provided by the coefficient matrices $[\mathbf{H}]_{\mathbf{r}}^{*}$ and $[\mathbf{G}]_{\mathbf{r}}^{*}$ and the contribution from the COD $\{\delta\}_{\mathbf{n}}$ of crack $n$ by the coefficients matrix $[\mathbf{D}]_{\mathbf{r n}}^{*}$. The determination of the boundary unknown displacement and traction components requires an additional system of boundary displacement equations

$$
\{\mathbf{u}\}=[\mathbf{H}]\{\mathbf{u}\}+[\mathbf{G}]\{\mathbf{t}\}+\sum_{\mathbf{n}=\mathbf{1}}^{\mathbf{N}}[\mathbf{D}]_{\mathbf{n}}\{\delta\}_{\mathbf{n}}
$$

where the contributions from the boundary displacement and traction are given by the matrices $[\mathbf{H}]$ and $[\mathbf{G}]$ and the contribution from crack $n$ by the matrix $[\mathbf{D}]_{\mathbf{n}}$. The system of equations obtained by assembling (25) and (26) is sufficient to determine the COD vectors $\{\delta\}_{\mathbf{n}}$ along with the unknown boundary displacement and traction components. Notice that the local contributions from each crack, given in terms of the local components, must be converted to the global components before adding them for all cracks. The solution comprises the unknown boundary stress and displacement components and the COD coefficients $\delta_{k}^{(m)}$ for each crack. Thus the stress intensity factors are calculated directly by the formula (24) in terms of $\delta_{k}^{(m)}$ without the additional post-processing.

\section{$6 \quad$ Numerical Results}

The coupling of the in-plane and the out-of-plane deformations is inherent in triclinic, monoclinic and trigonal systems of crystals. The orthorhombic, tetragonal, cubic and hexagonal systems also exhibit the coupling once the crystal is rotated out of the $x_{1} x_{2}$ symmetric plane. We select the cubic aluminum crystal, for which the two deformations are decoupled, and rotate the coordinate axes to produce a fully coupled compliance matrix. In the original orientation the crystal axes $a_{1}, a_{2}$ and $a_{3}$ of the cubic lattices are parallel to the coordinate axes $x_{1}, x_{2}$ and

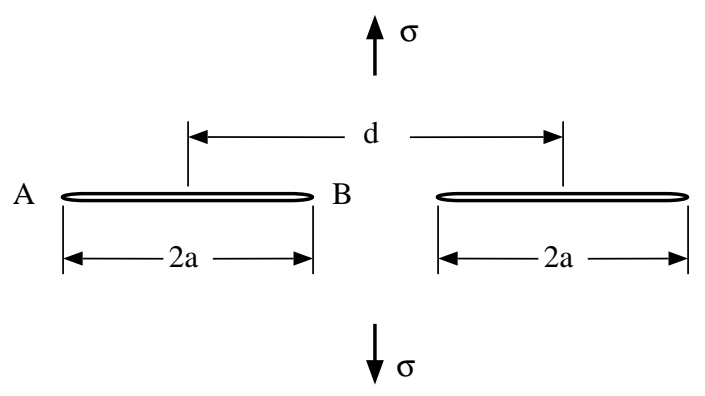

Figure 1: Two collinear cracks (with $2 a / d=0.9$ ) in an infinite body under uniaxial tension. 


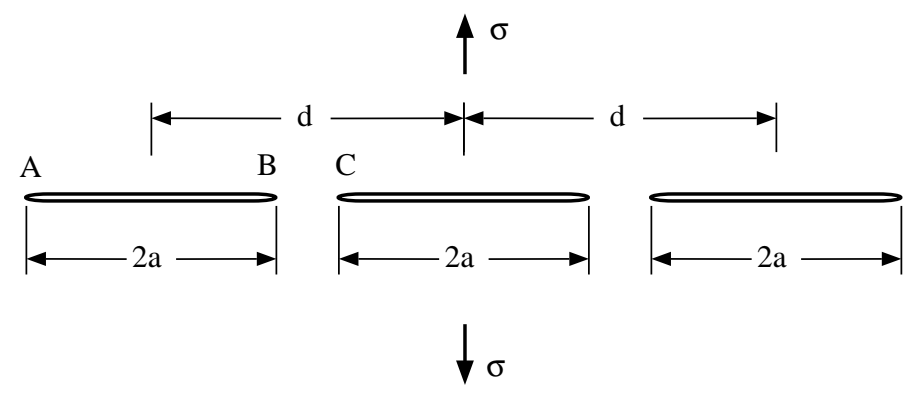

Figure 2: Three collinear cracks (with $2 a / d=0.9$ ) in an infinite body under uniaxial tension.

$x_{3}$, respectively. To achieve the general rotation of the coordinate axes we apply three consecutive rotations $[\theta, \psi, \phi]$ about the $x_{3}$-axis, the rotated $x_{2}$-axis and the rotated $x_{3}$-axis, respectively. For $\left[45^{\circ}, \arccos \frac{1}{\sqrt{3}}^{\circ}, 45^{\circ}\right]$ rotations, the compliance matrices of aluminum crystal (cubic) in the original and the rotated orientation are given by

$\mathbf{S}_{[0,0,0]}=\left[\begin{array}{cccccc}s_{11} & s_{12} & s_{13} & s_{14} & s_{15} & s_{16} \\ & s_{22} & s_{23} & s_{24} & s_{25} & s_{26} \\ & & s_{33} & s_{34} & s_{35} & s_{36} \\ & & & s_{44} & s_{45} & s_{46} \\ & & & & s_{55} & s_{56} \\ & & & & & s_{66}\end{array}\right]=\left[\begin{array}{cccccc}15.9 & -5.8 & -5.8 & 0 & 0 & 0 \\ & 15.9 & -5.8 & 0 & 0 & 0 \\ & & 15.9 & 0 & 0 & 0 \\ & & & 35.2 & 0 & 0 \\ & & & & 35.2 & 0 \\ & & & & & 35.2\end{array}\right]$,

$\mathbf{s}_{\left[45^{\circ}, \arccos \frac{1}{\sqrt{3}}^{\circ}, 45^{\circ}\right]}=\left[\begin{array}{cccccc}13.85 & -5.1167 & -4.4333 & 1.3667 & 1.3667 & 0 \\ & 13.85 & -4.4333 & -1.3667 & -1.3667 & 0 \\ & & 13.167 & 0 & 0 & 0 \\ & & & 40.667 & 0 & -2.7333 \\ & & & & 40.667 & 2.7333 \\ & & & & & 37.933\end{array}\right]$,

in units of $10^{-12} m^{2} / N$. Each matrix is symmetric and only the upper half is shown. Note that each crack configuration considered below is fixed with respect to the coordinate axes $x_{1}, x_{2}$ and $x_{3}$; only the compliance matrices are changed. We compare the SIF results with those obtained by the contour integral technique (Denda [6]) which will be enclosed in parentheses in the listings.

All problems of cracks in an infinite domain below have actually been obtained for a finite domain large enough, compared to the crack size, to be considered as infinite. The number of Chebyshev polynomials used for each crack is ten $(M=10$ in $(16))$. This is the number required to produce the extreme accuracy comparable to that obtained by the contour integral technique (Denda [6]). For 
routine calculation, however, the use of seven terms is good enough. Table. 1 shows the stress intensity factor (SIF) results for a center crack in an infinite body under remote tension $\left(\sigma_{22}\right)$ and out-of-plane shear $\left(\tau_{23}\right)$ applied separately, each of which produces only $K_{I}$ and only $K_{I I I}$. Values of $K_{I I}$ and $K_{I I I}$ for the tension and of $K_{I}$ and $K_{I I}$ for the out-of-plane shear, not listed in the table, are zero up to the fifth decimal point. The numerical results are in an excellent agreement with the normalized theoretical value of 1.0 for each case.

We look at two (Fig. 1) and three (Fig. 2) collinear cracks in an infinite body next. For these configurations it is known (Denda [6]) that the three modes are decoupled, under a single mode loading, and that each SIF is independent on the elastic constants of the solid. Thus, the handbook results, available only for the isotropic materials, can be used for comparison. Table 2 (two collinear cracks ) and 3 (three collinear cracks) list $K_{I}$ values under the uniaxial tension $\sigma_{22}$ for the two compliance matrices (27) and (28) as well as the handbook (Murakami et al. [15]) values for the isotropic solids. Values of $K_{I I}$ and $K_{I I I}$, not listed in the tables, are zero up to the fourth decimal point (i.e., 0.0000). For the two collinear crack problems accuracy of the handbook [15] $K_{I}$ values is reported to be $0.5 \%$. Note that for the three collinear crack problem, typical deviation from the handbook value is $0.1 \%$. Based on these comparisons, the error of the SIF results by the proposed singular crack element is estimated to be less than $1 \%$ for these simple problems, which is expected to hold for more complex problems for which no analytical results exist.

Table 1: Stress intensity factors of a center crack in an infinite plate under in-plane tension $\sigma_{22}$ and out-of-plane shear $\tau_{23}$, separately applied. Values in parentheses are taken from Denda [6].

\begin{tabular}{|l||c|c|}
\hline & $\mathbf{s}_{[0,0,0]}$ & $\mathbf{s}_{\left[45, \arccos \frac{1}{\sqrt{3}}, 45\right]}$ \\
\hline$K_{I} / \sigma \sqrt{\pi a}$ & $1.00011(0.99997)$ & $1.00011(0.99997)$ \\
\hline$K_{I I I} / \tau \sqrt{\pi a}$ & $1.00016(0.99989)$ & $1.00016(0.99989)$ \\
\hline
\end{tabular}

Table 2: Stress intensity factors for two collinear cracks (Fig. 1 with $2 a / d=0.9$ ) in an infinite body under tension $\sigma$.

\begin{tabular}{|c||c|c|c|}
\hline & $\mathbf{s}_{[0,0,0]}$ & $\mathbf{s}_{\left[45, \arccos \frac{1}{\sqrt{3}}, 45\right]}$ & Ref. [[15]] \\
\hline$K_{I A} / \sigma \sqrt{\pi a}$ & $1.11619(1.11686)$ & $1.11619(1.11687)$ & 1.11741 \\
\hline$K_{I B} / \sigma \sqrt{\pi a}$ & $1.44981(1.44950)$ & $1.44983(1.44951)$ & 1.45387 \\
\hline
\end{tabular}

Table 3: Stress intensity factors for three collinear cracks (Fig. 2 with $2 a / d=0.9)$ in an infinite body under tension $\sigma$.

\begin{tabular}{|c||c|c|c|}
\hline & $\mathbf{S}_{[0,0,0]}$ & $\mathbf{s}_{\left[45, \arccos \frac{1}{\sqrt{3}}, 45\right]}$ & Ref. $[[15]]$ \\
\hline$K_{I A} / \sigma \sqrt{\pi a}$ & $1.16393(1.16319)$ & $1.16393(1.16319)$ & 1.16439 \\
\hline$K_{I B} / \sigma \sqrt{\pi a}$ & $1.55853(1.55867)$ & $1.55853(1.55867)$ & 1.56454 \\
\hline$K_{I C} / \sigma \sqrt{\pi a}$ & $1.60016(1.60073)$ & $1.60016(1.60073)$ & 1.60685 \\
\hline
\end{tabular}


In the next three examples the SIFs for multiple cracks depend on the elastic constants. Table 4 gives the SIFs for two aligned parallel cracks (Fig. 3 ) subject to the uniaxial tension. The stress intensity factors for the three aligned parallel cracks (Fig. 4), and two inclined cracks (Fig. 5) are given in Tables 5 and 6, respectively. These SIF values are in an excellent agreement with those by the contour integral technique (Denda [6]) which serve as the only available source of comparison. For cracks in finite bodies the SIF results are available only for the isotropic solids. For such crack problems, Denda and Dong [10]'s whole crack singular element for the isotropic solids, based on the same Chebyshev polynomial interpolation of the COD as (16), has demonstrated an excellent agreement of the SIF results with the handbook values for the isotropic solids. Since Denda and Dong's WCSE for the isotropic solids is the subset of the proposed WCSE for the anisotropic solids, we expect the same reliable performance of the latter for cracks in finite bodies.

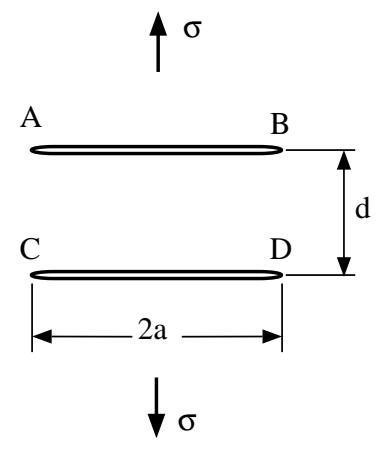

Figure 3: Two aligned parallel cracks (with $2 a / d=5.0$ ) in an infinite body under uniaxial tension.

Table 4: Stress intensity factors for aligned two parallel cracks (Fig. 3 with $2 a / d=5.0)$ in an infinite body under tension $\sigma$.

\begin{tabular}{|l|l||r|r|}
\hline & & $\mathbf{s}_{[0,0,0]}$ & $\mathbf{s}_{\left[45, \arccos \frac{1}{\sqrt{3}}, 45\right]}$ \\
\hline \multirow{3}{*}{$K_{I} / \sigma \sqrt{\pi a}$} & $\mathrm{~A}$ & $0.72223(0.72232)$ & $0.72399(0.72301)$ \\
\cline { 2 - 4 } & $\mathrm{B}$ & $0.72223(0.72232)$ & $0.72205(0.72234)$ \\
\cline { 2 - 4 } & $\mathrm{C}$ & $0.72223(0.72232)$ & $0.72205(0.72234)$ \\
\cline { 2 - 4 }$K_{I I} / \sigma \sqrt{\pi a}$ & $\mathrm{D}$ & $0.72223(0.72232)$ & $0.72399(0.72301)$ \\
\hline & $\mathrm{A}$ & $0.17089(0.17051)$ & $0.16348(0.16357)$ \\
\cline { 2 - 4 } & $\mathrm{B}$ & $-0.170789(-0.17051)$ & $-0.16364(-0.16346)$ \\
\cline { 2 - 4 } & $\mathrm{C}$ & $-0.170789(-0.17051)$ & $-0.16364(-0.16346)$ \\
\cline { 2 - 4 }$K_{I I I} / \sigma \sqrt{\pi a}$ & $\mathrm{D}$ & $0.17089(0.17051)$ & $0.16348(0.16357)$ \\
\cline { 2 - 4 } & $\mathrm{B}$ & $0.00000(0.00000)$ & $-0.00745(-0.00755)$ \\
\cline { 2 - 4 } & $\mathrm{C}$ & $0.00000(0.00000)$ & $-0.00978(-0.00998)$ \\
\cline { 2 - 4 } & $\mathrm{D}$ & $0.00000(0.00000)$ & $0.00978(0.00998)$ \\
\hline
\end{tabular}




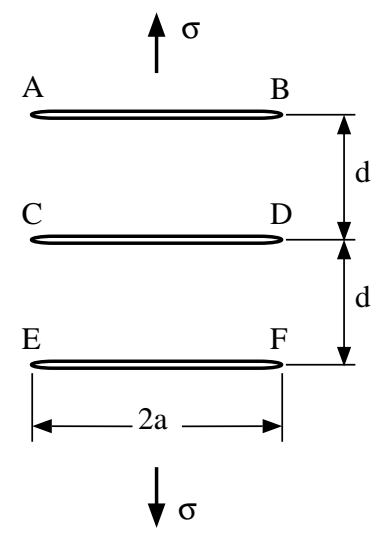

Figure 4: Three aligned parallel cracks (with $2 a / d=0.8$ ) in an infinite body under uniaxial tension.

Table 5: Stress intensity factors for aligned three parallel cracks (Fig. 4 with $2 a / d=0.8)$ in an infinite body under tension $\sigma$.

\begin{tabular}{|l|l||r|r|}
\hline & & $\mathbf{S}_{[0,0,0]}$ & $\mathbf{s}_{\left[45, \arccos \frac{1}{\sqrt{3}}, 45\right]}$ \\
\hline \multirow{5}{*}{$K_{I} / \sigma \sqrt{\pi a}$} & $\mathrm{~A}$ & $0.86077(0.86095)$ & $0.84992(0.85020)$ \\
\cline { 2 - 4 } & $\mathrm{B}$ & $0.86077(0.86095)$ & $0.84866(0.84894)$ \\
\cline { 2 - 4 } & $\mathrm{C}$ & $0.76839(0.76879)$ & $0.75112(0.75169)$ \\
\cline { 2 - 4 } & $\mathrm{D}$ & $0.76839(0.76879)$ & $0.75112(0.75169)$ \\
\cline { 2 - 4 } & $\mathrm{E}$ & $0.86077(0.86095)$ & $0.84866(0.84894)$ \\
\cline { 2 - 4 } & $\mathrm{F}$ & $0.86077(0.86095)$ & $0.84992(0.85020)$ \\
\hline \multirow{5}{*}{$K_{I I} / \sigma \sqrt{\pi a}$} & $\mathrm{~A}$ & $0.04324(0.04314)$ & $0.04437(0.04427)$ \\
\cline { 2 - 4 } & $\mathrm{B}$ & $-0.04324(-0.04314)$ & $-0.04476(-0.04466)$ \\
\cline { 2 - 4 } & $\mathrm{C}$ & $0.00000(0.00000)$ & $-0.00036(-0.00036)$ \\
\cline { 2 - 4 } & $\mathrm{D}$ & $0.00000(0.00000)$ & $-0.00036(-0.00036)$ \\
\cline { 2 - 4 } & $\mathrm{E}$ & $-0.04324(-0.04314)$ & $-0.04476(-0.04466)$ \\
\cline { 2 - 4 } & $\mathrm{F}$ & $0.04324(0.04314)$ & $0.04437(0.04427)$ \\
\hline & $\mathrm{A}$ & $0.00000(0.00000)$ & $0.00151(0.00151)$ \\
\cline { 2 - 4 } & $\mathrm{B}$ & $0.00000(0.00000)$ & $-0.01086(-0.01084)$ \\
\cline { 2 - 4 } & $\mathrm{C}$ & $0.00000(0.00000)$ & $0.00974(0.00972)$ \\
\cline { 2 - 4 } & $\mathrm{D}$ & $0.00000(0.00000)$ & $-0.00974(-0.00972)$ \\
\cline { 2 - 4 } & $\mathrm{E}$ & $0.00000(0.00000)$ & $0.01086(0.01084)$ \\
\cline { 2 - 4 } & $\mathrm{F}$ & $0.00000(0.00000)$ & $-0.00151(-0.00151)$ \\
\hline
\end{tabular}




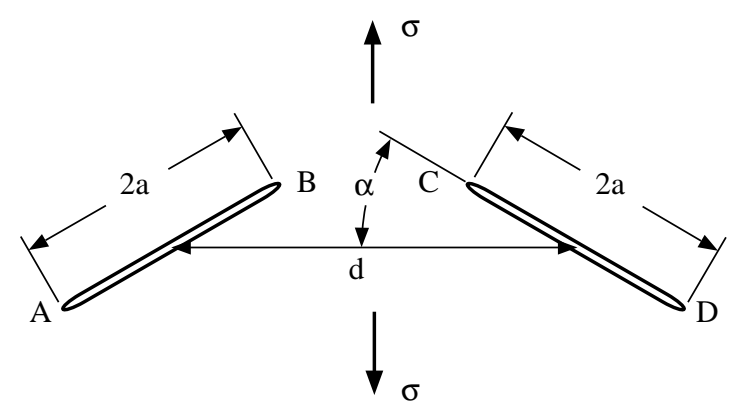

Figure 5: Two inclined cracks (with $\alpha=30^{\circ}$ and $2 a / d=0.9$ ) in an infinite body under uniaxial tension.

Table 6: Stress intensity factors for two inclined cracks (Fig. 5 with $\alpha=30^{\circ}$ and $2 a / d=0.9)$ in an infinite body under tension $\sigma$.

\begin{tabular}{|l|l||r|r|}
\hline & & $\mathbf{S}_{[0,0,0]}$ & $\mathbf{s}_{\left[45, \arccos \frac{1}{\sqrt{3}}, 45\right]}$ \\
\hline \multirow{3}{*}{$K_{I} / \sigma \sqrt{\pi a}$} & $\mathrm{~A}$ & $0.78779(0.78764)$ & $0.78779(0.78780)$ \\
\cline { 2 - 4 } & $\mathrm{B}$ & $0.91131(0.90907)$ & $0.91131(0.91105)$ \\
\cline { 2 - 4 } & $\mathrm{C}$ & $0.91131(0.90907)$ & $0.91131(0.91118)$ \\
\cline { 2 - 4 } & $\mathrm{D}$ & $0.78779(0.78764)$ & $0.78779(0.78780)$ \\
\hline \multirow{3}{*}{$K_{I I} / \sigma \sqrt{\pi a}$} & $\mathrm{~A}$ & $0.47394(0.47370)$ & $0.47394(0.47454)$ \\
\cline { 2 - 4 } & $\mathrm{B}$ & $0.45520(0.45407)$ & $0.45520(0.45355)$ \\
\cline { 2 - 4 } & $\mathrm{C}$ & $-0.45520(-0.45407)$ & $-0.45520(-0.45345)$ \\
\cline { 2 - 4 } & $\mathrm{D}$ & $-0.47394(-0.47370)$ & $-0.47394(-0.47452)$ \\
\hline \multirow{3}{*}{$/ I I$} & $\mathrm{~A}$ & $0.00000(0.00000)$ & $0.00033(-0.00032)$ \\
\cline { 2 - 4 } & $\mathrm{B}$ & $0.00000(0.00000)$ & $0.00171(0.00176)$ \\
\cline { 2 - 4 } & $\mathrm{C}$ & $0.00000(0.00000)$ & $-0.00042(-0.00039)$ \\
\cline { 2 - 4 } & $\mathrm{D}$ & $0.00000(0.00000)$ & $0.00084(0.00084)$ \\
\hline
\end{tabular}

For the contour integral technique (Denda [6]), the majority of the CPU time is spent for the contour integral evaluation in the post-processing. The proposed WCSE technique obtains the SIF in the main processing along with the boundary displacement and traction and no post-processing is required. The saving in the CPU is significant. Since the accuracy of the SIF results by the two techniques is made comparable by a judicious selection of the number of Chebyshev polynomials, the proposed WCSE approach has a clear advantage over the contour integral approach.

\section{Concluding Remarks}

We have developed the whole crack singular element for the straight crack in the 2 -D general anisotropic solids. It is simple but has the $\sqrt{r}$ COD and the $1 / \sqrt{r}$ 
stress singularity built in at its tips. In the BE solution, the stress intensity factors are determined, along with the unknown boundary displacements and tractions, without the post-processing. The proposed WCSE drastically simplifies the mixed mode analysis of multiple straight cracks in the general anisotropic solids with no sacrifice of the accuracy. Applications to multiple crack problems involving an extremely large number of straight cracks is now possible with this technique.

\section{Acknowledgment}

E. Mattingly was supported by the NSF IGERT Grant under subcontract from the University of Tennessee, Knoxville.

\section{References}

[1] Sollero, P. and Aliabadi, M. H., Fracture mechanics analysis of anisotropic plates by the boundary element method, Int. J. Fracture, vol. 64(4), pp. 269-284, 1993.

[2] Denda, M., A dislocation and point force approach to the boundary element method for mixed mode crack analysis of plane anisotropic solids, J. Chinese Institute of Engineers, vol. 22(6), pp. 677-693, 1999.

[3] Ang, W. T. and Clements, D. L., A boundary element method for determining the effect of holes on the stress distribution around a crack, Int. J. Numerical Methods Engng., vol. 23, pp. 1727-1737, 1986.

[4] Berger, J. R. and Tewary, V. K., Boundary integral equation formulation for interface cracks in anisotropic materials, Int. J. Numerical Methods Engng., vol. 20, pp. 261-266, 1997.

[5] Tan, C. L., Gao., Y. L., and Afagh, F. F., Boundary element analysis of interface cracks between dissimilar anisotropic materials, Int. J. Solids Structures, vol. 29(24), pp. 3201-3220, 1992.

[6] Denda, M., Mixed mode I, II and III analysis of multiple cracks in plane anisotropic solids by the BEM: a dislocation and point force approach, Int. J. of Engng. Anal. with Boundary Elements, vol. 25(4-5), pp. 267-278, 2001.

[7] Tan, C. I. and Gao, Y. L., Boundary element analysis of plane anisotropic bodies with stress concentrations and cracks, Composite Structures, vol. 20, pp. $17-28,1992$.

[8] Snyder, M. D. and Cruse, T. A., Boundary integral equation analysis of cracked anisotropic plates, Int. J. Frac., vol. 11, pp. 315-328, 1975.

[9] Chen, F. H. K. and Shield, R. T., Conservation laws in elasticity of the Jintegral type, J. Appl. Math. Physics (ZAMP), vol. 28, pp. 1-22, 1977. 
[10] Denda, M. and Dong, Y. F., Complex variable approach to the BEM for multiple crack problems, Compt. Methods Appl. Mech. Engrg., vol. 141, pp. 247-264, 1997.

[11] Lekhnitskii, S. G., Theory of Elasticity of an Anisotropic Elastic Body, Hoden-Day, San Francisco, 1963.

[12] Eshelby, J., Read, W., and Shockley, W., Anisotropic elasticity with applications to dislocation theory, Acta Metall., vol. 1, pp. 251-259, 1953.

[13] Altiero, N. J. and Gavazza, S. D., On a unified boundary-integral equation method, J. of Elasticity, vol. 10(1), pp. 1-9, 1980.

[14] Eshelby, J. D., The elastic field of a crack extending non-uniformly under general anti-plane loading, J. of Mech. Phys. Solids, vol. 17, pp. 177-199, 1969.

[15] Murakami et al., Y., Stress Intensity Factor Handbook, Pergamon Press, Oxford, 1987. 